\title{
Pulsed Gas Jets for Formation of High-Intensity Cluster Beams
}

\author{
N. G. Korobeishchikov, A. E. Zarvin, V. V. Kalyada, A. A. Schmakov \\ Department of Applied Physics, Novosibirsk National Research State University, Novosibirsk, Russia \\ Email: korobei@ci.nsu.ru
}

Received 2012

\begin{abstract}
The possibility of using of pulsed supersonic gas jets for the formation of high intensity cluster ion beams are discusses. The results of experimental investigations of pulsed gases expansion are generalized in terms of dimensionless similarity parameters. The results of the experimental study of formation of an high-intensity cluster beam of argon are presented. The fundamental phenomenon influences on the main parameters of cluster beam (cluster size, intensity ect.) are considered.
\end{abstract}

Keywords: Pulsed Supersonic Jet; Steady-Flow Region; Settling Time; Condensation of Gas; Cluster Beam

\section{Introduction}

Today the beams of atomic or molecular ions are in fact become an integral part of many modern technologies [1]. All conventional ion beam technologies are based on the individual collisions (or a cascade of binary collisions) of incident ions with nearsurface atoms. A completely different situation occurs during a collision the energetic large clusters with the surface. In this case, nearly simultaneous interaction of many particles of cluster around the same number of atoms in a solid occurs. This leads to deposition a high-energy density into a very small volume of the target material, and strong nonlinear effects: lateral sputtering, dry etching, and shallow implantation. Recently it was shown that the accelerated gas cluster ion beams have a number of unique advantages that allow them to be considered a promising basis for new technologies, including nanotechnologies [2,3].

It's known that in supersonic jet due to free expansion the gas temperature drops to cryogenic values the process of condensation occur and clusters can form [4]. A cluster can consist of a large number of identical or different particles and be in a different state of matter [5]. Forces, holding molecules in the cluster, could effect on the threshold energy of activation and ionization of molecules in a clusters, and result to broadening of energy levels. Large particle residence time increases the probability and intensity of energy exchange in a cluster that allows you to talk about "cluster catalysis" [6-7].

Since the course of condensation is determined by the number of particle collisions during expansion, you must increase the gas flow rate from the source, what requires a proportionate growth in the pumping performance of funds. Pulsed gas-jet sources allow you to succesfully solve this problem. The main advantage of pulsed sources compare to continuous flow devices is the high economic efficiency, explained by their relatively smaller dimensions, less stringent requirements on pumping systems, and lower consumption of high-cost materials. In addition, pulse sources easily compatible with powerful pulse activation systems: laser, discharge, etc.

\section{Dynamics of Gas Pulsed Expansion}

Pulse regime assumes that the gas source operates for a finite time with a certain sequence (frequency and pulse ratio). Therefore, the most important issue when using pulse source is the formation at required distance from the source the flow with sufficient duration and specified parameters, which similar steady-flow expansion.

Obviously the dynamics of free gas expansion is determined by the ratio of the momentum of the expanding gas to that of the background gas. Depending on stagnation pressure $\mathrm{P}_{0}$ and residual (background) pressure $\mathrm{P}_{\mathrm{b}}$ we may distinguish three principal regimes of supersonic pulsed expansion [8]:

a) expansion into a region of very low background pressure (expansion into vacuum),

b) expansion into a continuous medium (flooded space),

c) expansion into a region with reduced background gas pressure (intermediate case).

The regime (a) requires the maintenance of a low background pressure $\left(\mathrm{P}_{\mathrm{b}}<10^{-2} \mathrm{~Pa}\right)$. Other limiting regime $\left(\mathrm{c}, \mathrm{P}_{\mathrm{b}}>1\right.$ $\mathrm{Pa}$ ) applies only for expansion of a chock-wave-heated gas. For practical applications most important conditions correspond to regime (b, $1<\mathrm{P}_{\mathrm{b}}<10^{-2} \mathrm{~Pa}$ ). The relatively high background pressure allows the nozzle to operate at a large flow rate and form supersonic flow with developed relaxation processes. We have experimentally investigated the gasdynamic parameters of pulsed expansion of different gases $\left(\mathrm{He}, \mathrm{Ar}, \mathrm{N}_{2}\right)$ from sonic nozzles with diameters $\mathrm{d}=1 \mathrm{~mm}$ and $\mathrm{d}=0.5 \mathrm{~mm}$. The main parameters of pulsed jet are settling time of pulsed jet and duration of steady-flow region.

\subsection{Settling Time of Pulsed Jet}

The settling time of a nonsteady jet is defined as the time interval from the moment of opening of the source to the moment of establishment of steady flow parameters at a given distance from the nozzle. The settling time of a free jet is determined by motion of the front part of the nonsteady flow, which depends 
on the interaction of the expanding gas with the background gas, namely depends on the ratio of the momentum of the expanding gas to that of the background gas displaced from the flow region. Under expansion into vacuum (regime a) the leading front of expansion gas move with the limiting velocity $\mathrm{V}_{\mathrm{mn}}$ of nonsteady flow:

$$
V_{m m}=a *(\gamma+1) /(\gamma-1)
$$

where $a^{*}$ is sound velocity in the critical cross section of the nozzle. But settling time is determined by the maximum velocity of steady flow $V_{\text {ms }}$, which is depend on the total enthalpy $h_{0}$ of this gas:

$$
V_{m s}=\sqrt{2} h=a * \sqrt{(\gamma+1)} / \sqrt{(\gamma-1)}
$$

It was found that under expansion into a region with reduced background pressure (regime c) the retarding action of the background gas leads to that the leading front of expansion gas and the front of steady-flow region are the same. The boundary of a pulsed jet propagates with a velocity significantly smaller than the limiting steady-state value $\mathrm{V}_{\mathrm{ms}}$ for a given gas [8].

In order to generalize the experimental data, we used dimensionless parameters including characteristics of the expanding gas and the background gas, which play the role of similarity criteria [9]. Using this date, it's possible to calculate the settling time of pulsed jet for actual conditions.

\subsection{Duration of Steady-Flow Region}

At moment of switch off gas source, there arises a trailing (secondary) rarefaction wave characterized by nonsteady flow. Propagation of this secondary rarefaction wave downstream from the nozzle determines the trailing front of the steady flow region. Therefore, a question naturally arises as to what is the time of existence (or duration) of the steady flow at a given distance from a pulsed jet source.

It was established that the length (duration) of the steady flow region in a pulsed jet at a fixed distance from the source is independent of the ratio of heat capacities of expanding gases and is determined by the pulse duration at the nozzle exit and the ratio of stagnation and background pressures. The time of

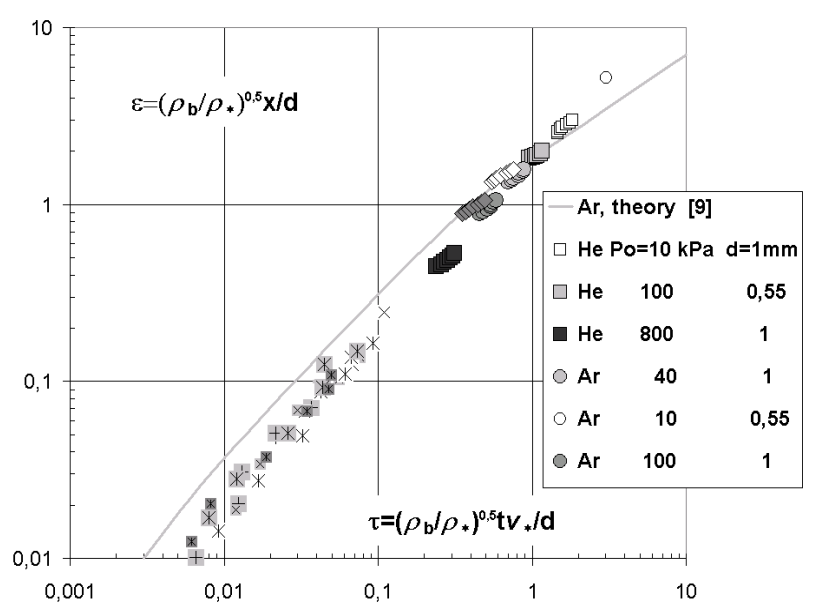

Figure 1. Generalized plot of settling time in dimensionless coordinates. existence of the steady state in a pulsed gas jet monotonically decreases downstream from the nozzle and drops with increasing background gas pressure due to the loss of particles in the leading and trailing rarefaction waves; this length increases with the initial momentum because the background gas is more intensively displaced from the flow region [8]. As a result, a situation is possible where the flow at a finite distance from the source does not attain a steady state even despite the fact that at the nozzle necessary conditions are satisfied.

\subsection{Formation of High Intensity Cluster Beams}

Using pulse source we carried out experimentally research on the formation of intensive cluster ions beams from the supersonic jets of Ar.

\section{Experimental}

The research was performed using the LEMPUS experimental setup of Novosibirsk State University [10]. The pulse valve with diameter of sonic nozzle $1 \mathrm{~mm}$ and the duration of gas pulse $\sim 1.3 \mathrm{~ms}$ was used. The stagnation pressure $\mathrm{P}_{0}$ varying from $1 \mathrm{kPa}$ before $10^{3} \mathrm{kPa}$. The measurements were performed by means of a molecular beam mass-spectrometer method [11-12].

\section{Results and Discussion}

To determine the optimal parameters for the formation of an intense cluster beam measurements of the total intensity of the neutral molecular flow were made by varying the stagnation pressure $\mathrm{P}_{0}$ and the distance of the nozzle - skimmer $\mathrm{x}_{\mathrm{ns}}$. The measurements were performed using a closed ionization pressure sensor Granville-Philips located on the axis of the molecular beam. Since the clusters are completely destroyed by collisions with the walls inside the sensor, the equilibrium density of the gas in the sensor is proportional to the intensity cluster molecular beam.

In Figure 3 shows the values of pressure on axis of molecular flow $\mathrm{P}_{\mathrm{a}}$ and background pressure in the ionizer section $\mathrm{P}_{\mathrm{b}}$, measured simultaneously at a fixed pressure $\mathrm{P}_{0}=5 * 10^{5} \mathrm{~Pa}$ at different nozzle-skimmer distances. Here and further the distance nozzle - skimmer is measured in caliber (diameter of the nozzle throat), $x / d_{*}$. There is an arrow shows the position of the boundary of the jet - direct shock wave (Mach disk), calculated from the known empirical formula:

$$
X_{M}=0.67 * \sqrt{P} / P_{k} .
$$

where $\mathrm{P}_{\mathrm{k}}$ is background pressure in the expansion chamber. Obviously, the background pressure in the ionizer chamber may influence the correctness of the sensor readings of the intensity of the molecular beam. Therefore, the true pressure in the molecular beam $\mathrm{P}_{\mathrm{mb}}$ defined as difference between $\mathrm{P}_{\mathrm{a}}$ and $\mathrm{P}_{\mathrm{b}}$.

When moving from the Mach disk to the nozzle increases leakage of gas through the skimmer into ionizer chamber according to the isentropic density distribution of gas in the jet ( $1 / x^{2}$, on the figure the dotted line). As a result, the background pressure $\mathrm{P}_{\mathrm{b}}$ increases proportionally. The pressure of the molecular beam $\mathrm{P}_{\mathrm{mb}}$ also dramatically increased in proportion to $1 / \mathrm{x}^{2}$ after leaving the Mach disk $\left(\mathrm{x} / \mathrm{d}_{*}<330\right)$. However, the 
increase in background pressure leads to molecular beam scattering, which leads initially (at $\mathrm{x} / \mathrm{d}_{*} \sim 240$ ) to a deviation from linear growth, and then (at $\left.\mathrm{x} / \mathrm{d}_{*}<200\right)$ and a pressure drop $\mathrm{P}_{\mathrm{mb}}$. Thus, for these conditions the molecular beam is formed with a maximum intensity at distances nozzle - skimmer 150-200 calibers.

Using this algorithm of measurements, we calculated the intensity of the clustering molecular beam for several fixed stagnation pressure (Figure 3). Despite the fact that the size of the jet (the distance to the Mach disk) changes markedly with increasing $\mathrm{P}_{0}$, the optimum distance of the nozzle - skimmer of about the same - 150-200 calibers. The maximum intensity ( $2 * 10^{18}$ molecules $/ \mathrm{sm}^{2} * \mathrm{sec}$ ) is attained at $\mathrm{P}_{0}=6 * 10^{5} \mathrm{~Pa}$.

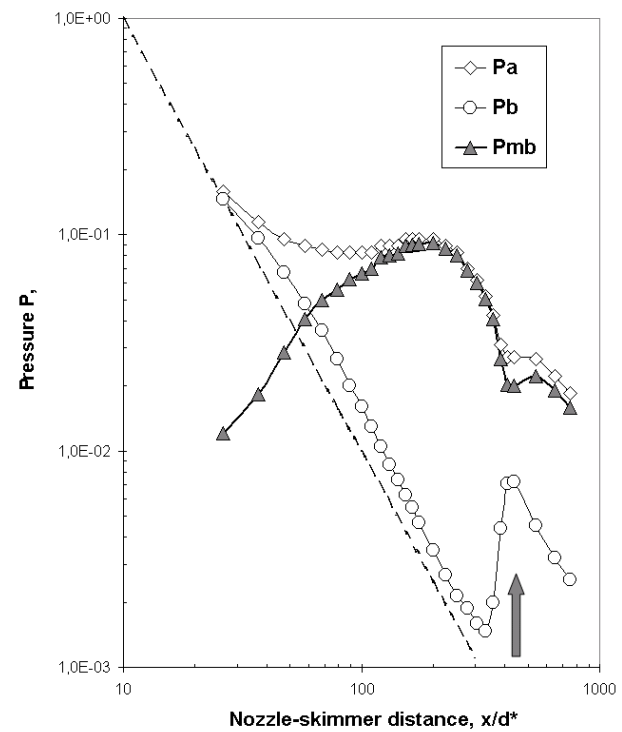

Figure 2. The pressure on axis of molecular flow, $P_{a}$, background pressure in the ionizer section $P_{b}$, and pressure in the molecular beam $P_{m b}$ depend of nozzle-skimmer distances at $P_{0}=5 * 10^{5} \mathrm{~Pa}$.

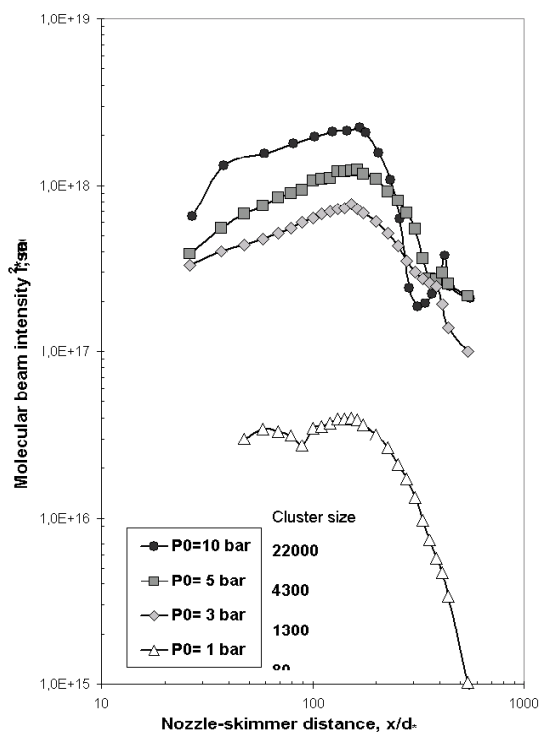

Figure 3. The intensity of cluster molecular beam (molecules/ $\mathrm{sm}^{2} *$ sec) depends of pressures $P_{0}$ and nozzle-skimmer distances.

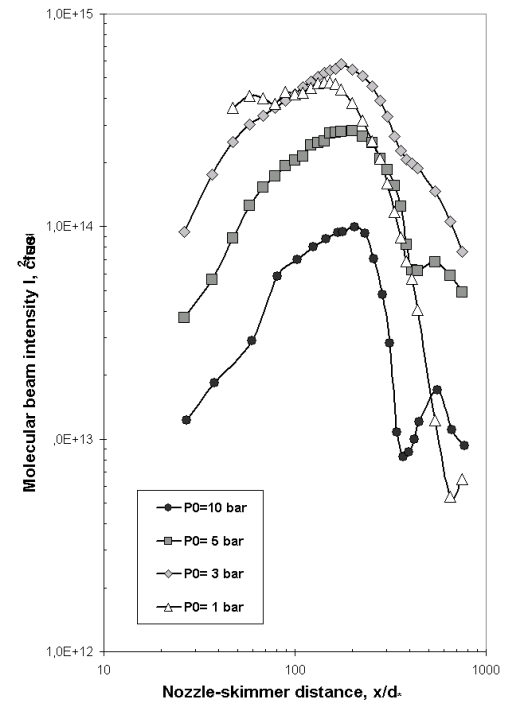

Figure 4. The intensity of cluster molecular beam depends of pressures $P_{0}$ and nozzle-skimmer distances.

It should be noted a significant non-linearity depending on the maximum intensity of the clustering molecular beam from the pressure $\mathrm{P}_{0}$ : at $\mathrm{P}_{0}=10^{5} \mathrm{~Pa}$, the maximum intensity of more than 15 times smaller than at $\mathrm{P}_{0}=3 * 10^{5} \mathrm{~Pa}$. One of the reasons is the influence of background gas in the expansion chamber. At low pressures transient regime of expansion formed with a blurring of shock waves limiting outside the core of the jet and, consequently, with a noticeable penetration of background gas, resulting in a decrease in the intensity of the molecular flow. In this case, at $\mathrm{P}_{0}=10^{5} \mathrm{~Pa}$, there is no central Mach disk (calculated position $-\mathrm{x} / \mathrm{d}_{*}=280$ ).

Another important reason is the various stages of condensation of gas flow in a supersonic flow. As you know, in a free jet heavy particles (in this case - clusters) are concentrated at the jet axis as a result of gas-dynamic separation [4]. Accordingly, the intensity of the beam is determined clustering molecular condensate fraction (fraction of gas in a bound state) and the size of the clusters. For our conditions, we calculated the dimensionless similarity parameters for flows with condensation, Hagena's parameter [13].

Using the mean cluster sizes intensities of the molecular beam were calculated in terms cluster $/ \mathrm{sm}^{2}$ sec, the results are shown in Figure 4. It is seen that, except for the very low pressure, with increasing $\mathrm{P}_{0}$ the flow of clusters on the axis of the jet decreases. This is explained by the previously mentioned factors - the proportion of output saturation and condensation on the continued growth of the average cluster size. Thus, the condensation at this stage continues, mainly due to consolidation of small clusters. So under such condition it is possible obtained a cluster beam very high intensity: up to $4-10^{14}$ clusters $/ \mathrm{sm}^{2} *$ sec.

As a result of experimental investigations the optimal conditions for the formation of a clustering molecular beam from supersonic jets of argon have been determined. A maximum intensity of $4 * 10^{14}$ clusters $/ \mathrm{cm}^{2} *$ sec for the clusters with an average size of 1000 molecules, and $8 * 10^{13}$ for clusters with an average size of more than 20,000 molecules have been reached. 


\section{REFERENCES}

[1] L.A. Giannuzzi and F.A. Stevens. Introduction to Focused Ion Beams: Instrumentation, Theory, Techniques and Practice. Springer Press. 2004.

[2] N.V. Popok and E.B. Campbell "Beams of atomic clusters: effect on impact with solid," Reviews of Advanced Materials Science, vol. 11, pp. 19-45, 2006.

[3] I. Yamada, «Cluster ion beam process technology - 20 years of R\&D history", Nuclear Instruments and Methods in Physics Research B, vol. 257, pp.632-638. 2007.

[4] H. Pauly, Atom, Molecule, and Cluster Beams II. Springer-Verlag, Berlin, 2000.

[5] Handbook of Nanophysics 2. Clusters and Fullerens. Ed. by Klaus D. Scattler. New York: CRC Press, 2011.

[6] V. Zh. Madirbaev, A. E. Zarvin, N. G. Korobeishchikov and R. G. Sharafutdinov, "Ion-cluster reactions initiated by an electron beam in mixtures of argon with methane and monosilane,” Phys. of the Solid State, vol. 44, pp. 515-517, 2002.

[7] V. Zh. Madirbaev and A. E. Zarvin, "On the possibility of a cluster-catalytic reactions for the synthesis of heavy hydrocarbons," Book of Abstracts of the $7^{\text {th }}$ Int. Seminar on Flame Structure and First Young Res. Sch. on Flame Study. Novosibirsk,
July 11-15, p. 56, 2011.

[8] N. G. Korobeishchikov, A. E. Zarvin and V. Zh. Madirbaev, "Hydrodynamics of pulsed supersonic underexpandet jets: Spatiotemporal characteristic”, Tech. Phys., vol. 49, pp. 973-981, 2004.

[9] S. F. Chekmarev and N. V. Stankus, "Gasdynamic model and similar relations for the starting process in supersonic nozzles and jets”, Sov. Tech. Phys., vol. 29, pp. 920-925, 1984.

[10] A. E. Zarvin, N. G. Korobeishchikov, V. Zh. Madirbaev, G. G. Gartvich, V. V. Kalyada and V. S. Airapetyan, "A universal small-sized vacuum installation for gas-kinetic investigations”, Instrum. Exp. Tech., vol. 43, pp. 641-649, 2000.

[11] N. G. Korobeishchikov, A. E. Zarvin, V. Zh. Madirbaev and R. G. Sharafutdinov, "Condensation of argon, monosilane and their mixture in a pulse free jet”, Plasma Chem. Plasma Proc., vol. 25, pp. 319-349, 2005

[12] A. E. Zarvin, N. G. Korobeishchikov, V. V. Kalyada and V. Zh. Madirbaev, "Formation of mixed clusters in a pulsed helium-oxygen-isoprene supersonic jet”, Eur. Phys. J. D, vol. 49, pp. 101-110, 2008.

[13] O.F. Hagena, “Cluster ion sources,” Review of Science Instruments, vol. 63, pp. 2374-2379, 1992. 